\title{
Surface acoustic waves-localized plasmon interaction in pillared phononic crystals
}

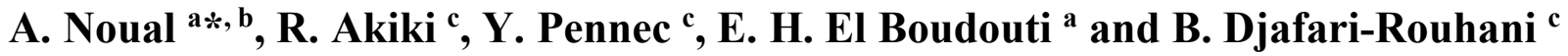 \\ ${ }^{a}$ LPMR, Département de Physique, Faculté des Sciences, Université Mohamed Premier, 60000 Oujda, Morocco. \\ ${ }^{b}$ Département de Physique, Faculté Pluridisciplinaire Nador, Université Mohamed Premier, 60000 Oujda, Morocco. \\ ${ }^{c}$ IEMN, UMR CNRS 8520, Département de Physique, Université de Lille, 59655 Villeneuve d'Ascq, France.
}

\begin{abstract}
We study numerically the interaction of surface acoustic waves propagating at the surface of a multilayer structure (the so-called Sezawa waves) with localized surface plasmons at the bottom of metallic pillars deposited on the substrate. The pillars are made of gold and the multilayer structure is constituted by a layer of gold on a silicon substrate and covered by a thin spacer dielectric material. We shall be interested by localized plasmons which are the analog of metal-dielectric-metal surface plasmons. The physical characteristics of these plasmons such as their eigenvalues, absorption or reflection spectra are modulated by the surface acoustic waves due to the deformation of the structure in the vicinity of the pillars. The surface waves are generated in the area in front of the pillars deposited on the substrate. Two types of acoustic modes are envisioned to interact with the localized plasmons, namely confined modes associated with the local resonances of the pillars and the propagating Sezawa modes that deform the structure in the vicinity of the surface. We discuss the efficiency of phonon-plasmon coupling for both types of modes and select those which can be useful for an experimental realization based on this platform. Also, we show that, besides the plasmon frequency, the phonon-plasmon coupling magnitude strength is sensitive to the distance between the pillars, revealing the role of interaction between the latters on the coupling. The geometrical parameters are chosen such that the acoustic waves are in the sub-GHz range and the plasmons around the telecommunication wavelength of 1.55 micron. This work may help designing new optomechanical devices that can be controlled by fast coherent acoustics, and facilitates applications invloving enhanced sound-light interactions such as light modulation.
\end{abstract}

Keywords: Surface acoustic waves, 3D Multi-layered structure, MIM-localized surface plasmon, phonon-plasmon coupling.

\section{Introduction}

Lately, a great deal of attention has been paid to the interaction of ultrahigh-frequency elastic waves with optical waves within nano or micro-scale photonic structures, with a view to manipulate/modulate the optical properties of the latters [1-5]. Such devices are termed as optomechanical structures, and the intense interest they raised relates to their utility in both fundamental physics as for controlling mechanical motion by light and quantum information processing, and their potential practical applications as sensors for high precision measurements of mass, charge or small distances [5-9]. Two physical mechanisms are involved in the coupling of acoustic phonons and light inside an homogeneous bulky material: i) the first one is due to the local stress field generated by the passage of the acoustic wave, known as the photoelastic-effect $[10,11]$; ii) the second mechanism is based on the perturbation by the elastic waves of the structural interfaces of the system, called interface moving-effect [12]. To achieve strong optomechanical interactions certain types of nano/mico-structures have been used lately, the so-called phoxonic crystals [13]. These structures can exhibit simultaneous band gaps for both photons and phonons, that is, they can support both light and sound localization within the same cavity, giving rise to enhanced photon-phonon coupling. Based on these crystal cavities, optomechanical (OM) interactions have been greatly investigated in 2D-crystals [14, 15], nanobeams [16-21] and in crystal slabs [22-25]. More recently, Aram and Khorasani studied optomechanical coupling strength in various triangularly shaped phoxonic crystal cavities [26], and Hsu et al have shown efficient generation of hybrid plasmonic-photonic band gap using acoustic Lamb waves [27]. On the other hand, phononplasmon coupling has been demonstrated in phoxonic-plasmonic slab cavities based on periodic metal strips [28], and also through the use of acoustic surface waves where strong optomechanical interactions in hybrid photonicplasmonic crystal nanocavities have been shown [29].

Besides, it is well established that localized surface plasmon (LSP) waves supported by small metallic nanoparticles give rise to a high degree of light confinement [30,31]. In particular, light intensity can be enhanced several orders of magnitude closer to the particle surface, in a much smaller length scale as compared to the light wavelength in vacuum. This outstanding property has been harnessed in a number of applications as for conceiving biosensors [32- 
34], in photothermal therapy [35, 36] and to achieve plasmon-induced enhancement of solar cells efficiency [37, 38]. Furthermore, coupling of LSP with vibrating nanoparticles of different shapes have been intensely studied; we can cite for instance the work of van Dijk et al [39] in which gold spherical nanoparticles were considered, or cubic particles [40], rods [41], columns [42] and antennas [43]. Generally, the interaction of LSP and mechanical oscillations sustained by metallic nanoparticles are studied via pump-probe experiments [44, 45], or based on Raman scattering [46-48]. Lately, Mrabti et al have [49] studied numerically the phonon-plasmon interaction in a system made of gold nano-cylinders coupled to an Au-film, and sitting on a Si membrane. We would like to indicate on the other hand, that the coupling of surface plasmons (or LSP) with phonons instead of photons enables further enhanced optomechanical interactions due to the fact that reachable or allowable (under the elastic limit) acoustic phonons amplitudes (of few nanometres) are in the same range of characteristic dimensions of the domain where the plasmons are oscillating or localized (i.e., in an optical cavity for instance) [29]. Such strong optomechanical interactions could lead to fundamental physical studies such as optical management of thermal properties of nanosystems in the quantum regime [50], enhanced optical nonlinearity [51] and optomechanically induced transparency [52]. Additionally, the use of acoustic surface waves (SAW) at the aim of giving rise to enhanced phonon-plasmon coupling such as in [29], constitutes an important step towards experimental realization of the proposed SAW-based device for strong optomechanical interactions. Indeed, nowadays SAW are experimentally easily generated using planar interdigital transducers and SAW-based components have been employed for a variety of applications already $[53,54]$. Nonetheless, except the work in [29], SAW-based devices for enhanced phonon-plasmon coupling are quite rarely reported in the literature.

Motivated by this context, we develop a new approach in this paper based on the use of surface acoustic waves (SAW) for the investigation of phonon-localized surface plasmons enhanced interactions in a novel multilayer structure. Firstly, we study the phononic and optical properties of a system consisting of a periodic array of gold nanocylindres (AuNCs) deposited on top of a multilayer structure where the latter is made of a silicon substrate supporting an Au-metal film covered by a thin polymer material acting as a spacer with respect to the pillars. Then, we investigate surface waves interaction with localized plasmons at the bottom of the pillars which are the analogs of metal-dielectric-metal-LSP (MIM-LSP) and are chosen to arise in the near infrared frequency range (around $1.55 \mu \mathrm{m}$ ). The region underneath the AuNCs where the MIM-LSP is confined acts as an effective MIM-cavity. Subsequently, elastic phonons deforming this cavity volume results in the modulation of the frequency of the resonating plasmonic mode. The structure exhibits two types of surface acoustic modes namely localized phonons confined in the pillars and propagating Sezawa waves near the surface. The interaction of these two types of modes with localized plasmon is investigated using moving interface effect. We demonstrate effective modulation of the plasmon frequency by certain Sezawa and localized phonons through the simulation of the system plasmon eigenfrequency versus elastic modes phase. We also show the role of interactions between the pillars on phonon-plasmon coupling magnitude strength. Localized and Sezawa phonons that strongly couple with the plasmon might be useful for the conception of experimentally realizable SAW devices (which are easily integrated on chip) based on the proposed platform, with a view to various potential applications such as ultrasensitive sensors and acousto-plasmonic modulators.

This paper is organized as follows: in section 2, we present full 3D-numerical simulations of the phononic properties of the periodic multilayer structure. In section 3 we focus on the optical properties of the system and then in section 4, we investigate Sezawa and localized phonons-plasmon coupling. In section 5, we study surface acoustic waves interaction with localized plasmon exhibited by the multilayer system where one row of gold pillars is placed on top. The conclusions are given in section 6 .

\section{Phononic properties}

A sketch of the studied structure is presented in Fig. 1(a); it consists in a square array of gold nanocylindres sited on a multilayered substrate composed out of a gold metal film, a thin polymer material (polyamide) acting as a spacer and a semi-infinite substrate made of silicon lying underneath. Throughout the paper, AuNCs have radius $R=$ $165 \mathrm{~nm}$ and height $h_{A u}=300 \mathrm{~nm}$, the thickness of the polymer spacer is chosen as $d_{p}=50 \mathrm{~nm}$, whereas the Aufilm is $D_{A u}=100 \mathrm{~nm}$ thick and the lattice parameter is set as $a=1000 \mathrm{~nm}$. The polymer material is an isotropic medium whose elastic properties are represented by Young modulus $E=2.5 \mathrm{GPa}$, Poisson ratio $v=0.34$ and its density $\rho=1400 \mathrm{~kg} / \mathrm{m}^{3}$. On the other side, gold is an anisotropic material with face centered cubic crystal structure and silicon crystallizes in a face centred diamond cubic structure; their elastic constants parameters $C_{11}, C_{12}$ 
and $C_{44}$ were taken from reference [11]. Numerical simulations in this paper are performed based on finite element method using commercial software Comsol Multiphysics.

(a)

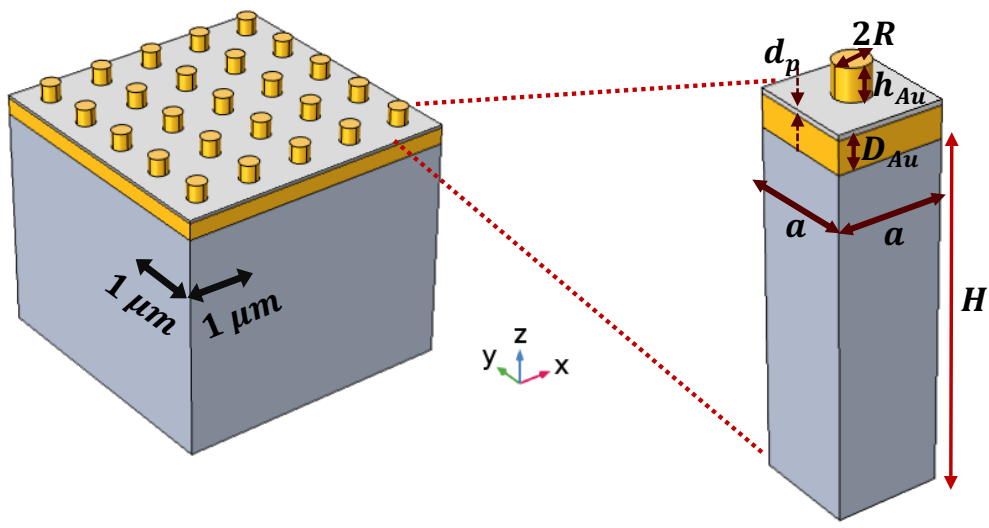

(b) (c)
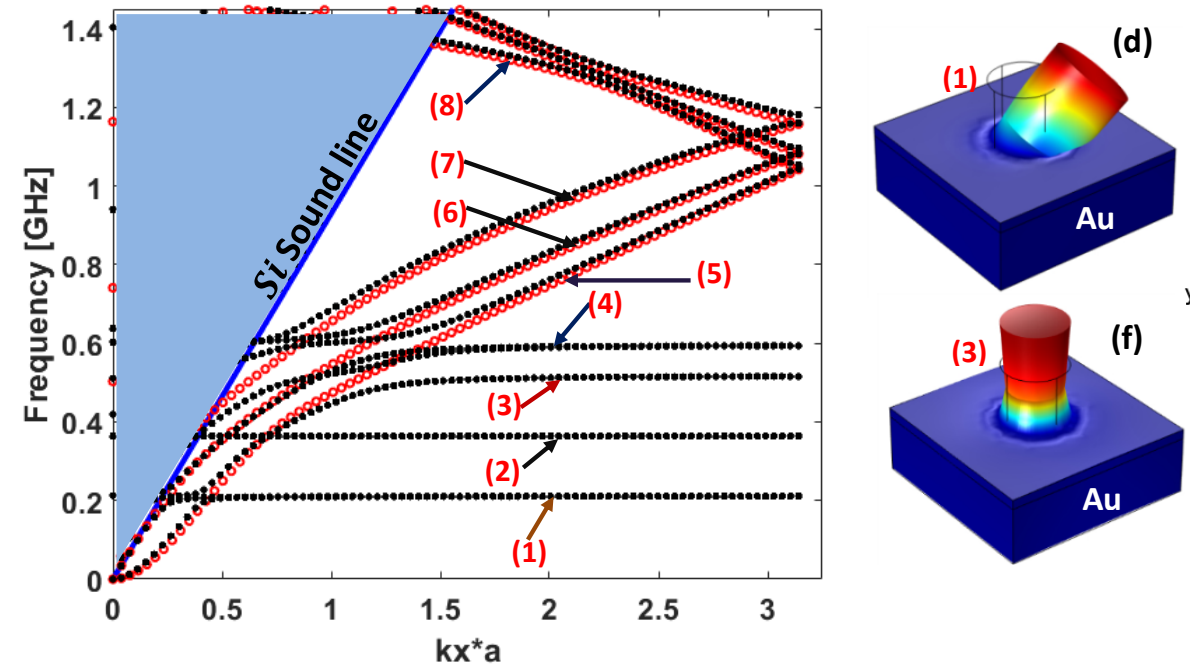

$y_{2}^{z} x$

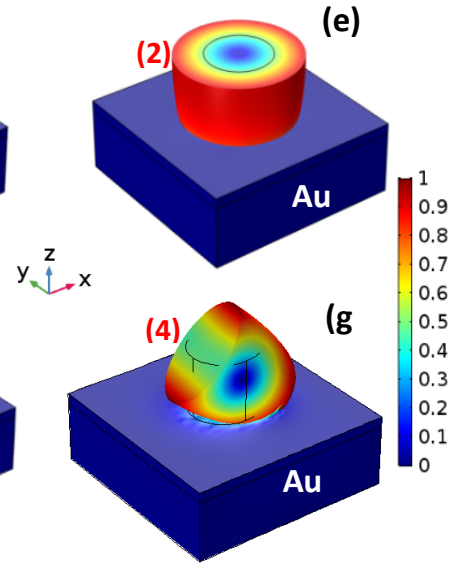

(6)

(i)

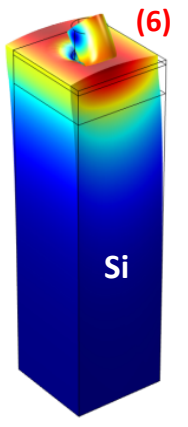

(j)

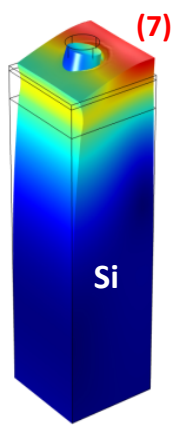

(k)

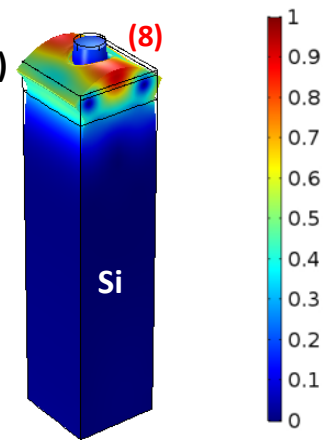

Fig. 1. (a) Schematic diagram of the studied structure. (b) Zoom-in on a unit cell of the structure indicating the shape parameters of the nanocylinder and of the multilayer substrate. (c) Simulated band diagram of the structure depicted in Fig. 1(a) in black dotted lines. Dispersion curves of the structure in absence of gold nanocylindres are also plotted in red circles. (d)-(g) Displacement field maps of the localized modes within the gold nanocylinders (the Si substrate where the filed vanishes for these modes is not shown), referred to as (1)-(4) in (a), respectively. (h)-(k) Same as in (d)-(g), but for the modes referred to as (5)-(8) in (a), which are localized in the Au-thick film near the surface of the device (Sezawa modes). Except for mode (8), depicted at $k_{x} a=1.68$, remaining modes are all given at the same reduced wave vector $k_{x} a=2$. Presented displacement fields maps are normalized with respect to their maximum value within the system. 
In particular, to get the band diagram of the structure we used solid mechanics module in Comsol with the following boundary conditions: Floquet periodic conditions are applied along $x$ and $y$ directions, free surface is set on top of the system whilst a low-reflection boundary is chosen at the bottom of the structure to mimic a semi-infinite substrate. Moreover, the thickness of the latter in the model is chosen large enough such as $H=10 a$ to realize accurately the condition of a semi-infinite substrate. Figure 1(c) shows the simulated band diagram of the system along the $\Gamma X$ direction. The four lower branches below the sound line in silicon are totally flat from $k_{x} a \geq 1.5$ onwards; these are eigen-modes of the gold nanocylinder. It is worth pointing out that the first and fourth branches are two times degenerate modes associated with flexural motions of the pillars. Above the four branches three other dispersive branches are apparent, resulting from the folding of the former at the Brillouin zone edge due to periodicity. These three branches arouse out of the interaction of Sezawa modes localized in the Au-thick film and the AuNCs localized modes, giving rise to the observed dispersive curves. To show this, we have plotted in small red squared markers in Fig. 1(c) the dispersion curves of the structure in absence of AuNCs, namely the RayleighSezawa modes. The latter reveals three branches that superimpose quite well with branches of pillared system (of Fig. 1(a)) in the middle and high frequency range. Their displacement fields (see below) indicate a strong localization in gold film underneath the surface. In Figs. 1(d)-1(g), are presented displacement field maps corresponding to the lower fourth eigen-modes localized in gold nanocylinders (branches (1)-(4) in Fig. 1(c)). On the other hand, in Figs. 1(h)-1(k) we give the filed maps corresponding to some of the modes originating from Sezawa branches, (identified as (5), (6), (7) and (8) in Fig. 1(c)). The two-fold degenerated modes (1) are a first order flexural mode of the cylinder along the $(O x)$ and $(O y)$ axes whose frequency is $\approx 0.2 \mathrm{GHz}$, mode (2) is a radial breathing mode (of the cylinder) at the frequency of $\approx 0.35 \mathrm{GHz}$, mode (3) is a compression mode where the cylinder gets compressed symmetrically towards its axis at the frequency $\approx 0.508 \mathrm{GHz}$. The compression is accompanied by an elongation movement along $z$-axis. The degenerated fourth modes $(4)$ are is a higher order flexural mode with the eigen-frequency $0.58 \mathrm{GHz}$. Displacement filed maps of modes $(5)(\approx 0.71 \mathrm{GHz}),(6)(\approx$ $0.83 \mathrm{GHz}),(7)(\approx 0.91 \mathrm{GHz})$ and $(8)(\approx 1.33 \mathrm{GHz})$ indicate that these are Sezawa modes confined within Au-film near the surface, and coupled to some extent with AuNCs modes. As a matter of fact, gold nanocylinder in modes (5), (6) and (7) are entrained in a flexural-like motion (Figs. 1(h)-1(j)) and in a compression/elongation-like motion in mode (8) (Fig. 1(k)). We are particularly interested in those eigen-modes that have a fraction of the associated elastic energy localized within or around the dielectric spacer, that is, inside the volume where MIM-LSP mode of the system shall be localized. This will result in their overlapping and hence possibly their coupling. In order to check out how the elastic energy of modes (1)-(8) is distributed inside the structure (except in the substrate where the field vanishes for these modes), especially within the dielectric spacer, we have plotted in the supplementary information (S.I.) in Figs. S1(a)-S1(h) the rate of the elastic strain energy density localization in a $z x$-cut plane of the structure. The former is defined as the ratio of the elastic strain energy density integrated over the spacer volume reported to the maximum value taken by the energy density within the volume of the whole structure, that is:

$$
E_{\text {loc }}=\frac{\iiint_{V_{\text {spacer }}} U d v}{\operatorname{Max}_{\text {Vtot }}(U)}
$$

where, $U$ is the strain energy density, it reads for an elastic anisotropic material as follows:

$$
U=\frac{1}{2} C_{i j k l} \varepsilon_{i j} \varepsilon_{k l}
$$

$C_{i j k l}$, being the components of the fourth order stiffness tensor of the material and $\varepsilon_{i j}$ (or $\varepsilon_{k l}$ ) are the strain tensor components. From Figs. S1(a)-1(h), one can note that modes (1) and (2) show a quite weak localization of the energy within the dielectric spacer as opposed to the rest of the modes; the compressional mode (3) indicates the higher localization rate $(\sim 30 \%)$. Sezawa surface mode (7) shows the second higher rate of localization and is followed by Sezawa modes (8), (5) and (6), respectively. High order flexion mode (4) manifests a comparable localization to mode (5). One notes that mode (8) shows some strain energy localisation near Si substrate, which simply relates to the deformation induced by this mode close to the interface of the Au-film and the silicon substrate underneath. Clearly, modes (3)-(8) seem to be good candidate for the acousto-optic interaction, subsequently it is important to get a more specific idea on how these modes might affect a resonating MIM-LSP mode within the dielectric spacer. To that end, we analyze in Figs. S1(i)-S1(t) the behavior of the real parts of the displacement field components along the $x$-axis (component $-u$ ) and along the $y$-axis (component- $-v$ ), associated with modes (3)-(8) in the dielectric spacer. The volume of the spacer layer situated right underneath the gold nanocylinder act as an effective cavity where a propagating surface plasmon (in a MIM-like structure) reflects off the cylinder bottom edges, resulting in plasmonic standing waves. The resonance wavelengths of the latters are sensitive to the (effective) cavity thickness (which is the spacer thickness) and its length, which matches the cylinder diameter. This means that elastic modes 
changing one of these dimensions or both of them shall modulate the resonance plasmon mode wavelength. Thus, it is essential to figure out how the studied modes deform the effective cavity volume during an acoustic period of time. According to Figs. S1(i)-S1(t), mode (3) significantly compresses/stretches out the MIM-cavity volume during an acoustic period in an isotropic manner, and as a result a strong coupling between the MIM-LSP and mode (3) is expected. Almost similarly Sezawa eigen-modes (7) and (8) stretch and compress the cavity along $x$ and $y-$ axes, leading to its volume deformation. Nonetheless, this deformation is not uniform as for mode (3) which means the coupling strength might be different comparing with mode (3). In mode (4), the cavity oscillates together with the cylinder with some volume deformation occurring, especially along $x$-axis; the deformation is much weaker comparing with modes (3) and (8), so should be the plasmon-phonon coupling magnitude. Sezawa mode (5) stretches the cavity mainly along the $x$-axis, resulting in a non-negligible volume deformation of the cavity and suggesting that a good phonon-plasmon interaction rate might be observed with this mode. A weak volume deformation of the effective cavity might be noticed according to $u$ and $v$ components maps of Sezawa mode (6); the deformation magnitude is indeed seemingly quite smaller (almost negligible) comparing with modes (7), (8) and (5), which hints at a quite lesser phonon-plasmon coupling strength.

\section{Plasmonic properties}

To simulate the optical properties of the system, a medium of incidence (air) is added on top of a unit cell of the structure as shown in Fig. 2(a). We consider a normally incident plane wave polarized such that its electric field oscillates along $(O x)$ axis. The wavelength domain of interest here lies around $1550 \mathrm{~nm}$ ([1440 nm-1720 nm]). The refractive index of the polymer material (Polyamide) is $n_{p}=1.7$, and gold metal is modeled based on LorentzDrude model.

(a)

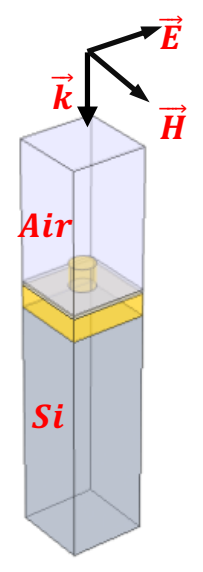

31

32 (b)
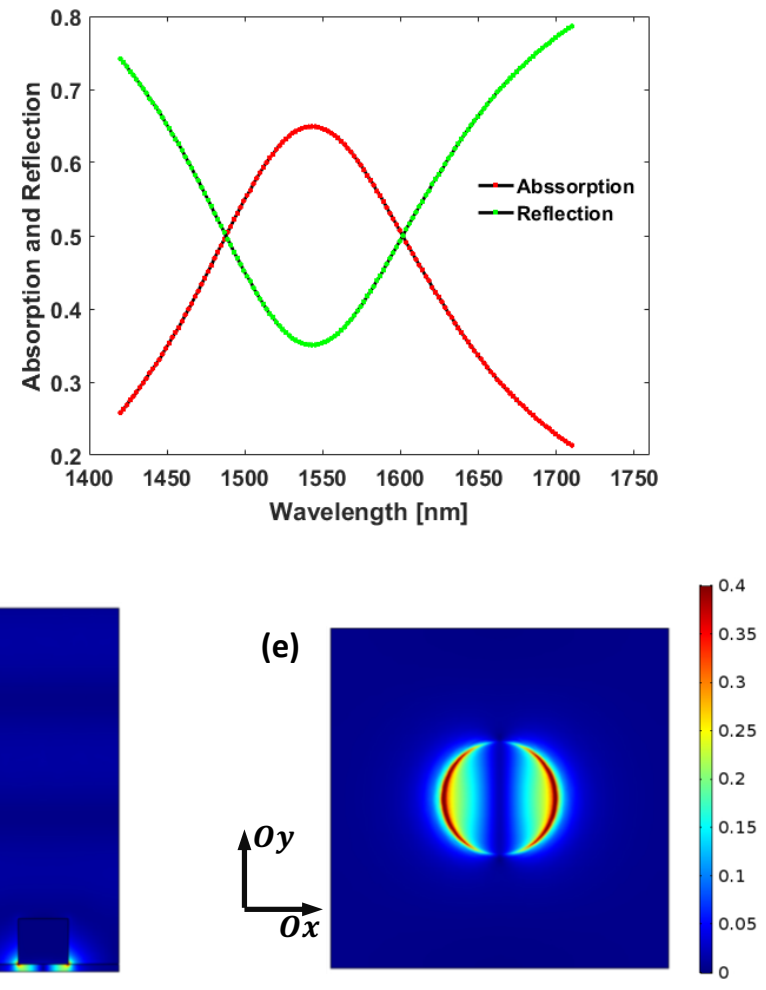

Fig. 2. (a) Sketch of a unit cell of the structure depicted in Fig. 1(a) on top of which an incidence medium made of air is added. A linearly polarized plane wave (E-field along $x$-axis) normally falling down on the structure is shown. (b) Corresponding simulated absorbance and reflectance spectra. (c) Electric field norm map plotted within the dielectric spacer and the Au nanocylinder. The E-field norm is normalized to its maximum value taken in the whole structure. (d)-(e) Same as in (c), but in a $z x$-cut plane of the geometry (median plane) and in the $x y$-plane lying right underneath the gold nanocylinder, respectively. 
Floquet-periodic boundary conditions are applied along $(O x)$ and $(O y)$ axes and port boundary conditions are set on the inlet (on top) and outlet (at bottom) of the structure. Fig. 2(b) gives the simulated absorbance and reflectance spectra of the structure, where an important absorption band extending from $\sim 1420 \mathrm{~nm}$ up to $\sim 1720 \mathrm{~nm}$ takes place; it reaches its higher rate of about $65 \%$ at the wavelength $\approx 1543 \mathrm{~nm}$. At the same time, the reflectance falls down at this same wavelength to around $35 \%$ indicating a strong localization of the light within or around the AuNC. The associated electric field norm distribution with the observed absorption peak is depicted within the polymer spacer and the AuNC in Fig. 2(c), where it can be seen that the lighwave is trapped down at the bottom of the pillar. The E-field distribution is also shown in the median $z x-$ plane of the structure and in the $x y-$ plane situated right underneath the AuNC, in Figs. 2(d) and 2(e), respectively. It is apparent that the excited mode is strongly localized within the dielectric spacer at the bottom of the AuNC, fitting the description of the so-called MIM-localized-surface plasmon (MIM-LSP) fairly well described in [49]. The effective cavity down below the AuNCs, where the standing plasmonic waves form will be called a MIM-cavity in the following. If the diameter of the gold particle is stretched to infinity, we end up with a unidimensional structure made out of silicon substrate (infinite)/ $\mathrm{Au}(300 \mathrm{~nm}) /$ polyamide polymer $(50 \mathrm{~nm}) / \mathrm{Au}(300 \mathrm{~nm}) / \mathrm{Air}$ (Fig. 3(a)). This system supports among (several) other modes MIM surface plasmon-polariton (MIM-SPP) modes confined within the polymer spacer [49], and which propagate with wave vector $k_{s p p}$ as shown in the inset of Fig. 3(a). The latter is sensitive to the dielectric spacer thickness and dependent on the incident light wavelength as well. We plotted in Fig. 3(a) the corresponding dispersion curve along with the light line in the polymer material. Based on Fig. 3(a), the effective index of the propagating surface plasmon is $n_{e f f}=k_{s p p} / k_{0} \approx 2.4204$ for a wavelength in vacuum equal to $1543 \mathrm{~nm}$ (resonance wavelength of the plasmon mode observed in Fig. 2). Along the interface on which the surface plasmon propagates the wavelength is about $\lambda_{s p p} \approx\left(2 \pi / k_{s p p}\right) \approx 637 \mathrm{~nm} \approx 2 D$, where $D=2 R$. Hence, the MIM-plasmon mode has typically a wavelength of the order of the cylinder diameter $(D=330 \mathrm{~nm})$, which provides a convincing explanation as to why plasmonic standing waves can form in the MIM-cavity. Furthermore, we depict in Fig. 3(b) the transverse $E_{z}$ component of the E-field map of a propagative MIM-SPP mode along with black arrows showing $E_{z}$-field lines, and in Fig. 3(c) we give the map of the transverse component of the E-field $E_{z}$ associated with the MIM-LSP (with black arrows showing lines of $E_{z}$ ) confined at MIM-cavity. The maps show a quite similar behavior of the transverse components of the electric fields in both cases, hinting at the origin of the observed plasmon mode in Fig. 2, that is, the reflection off the AuNCs bottom edges of propagating MIM-SPP modes, giving rise to resonating plasmonic standing waves.
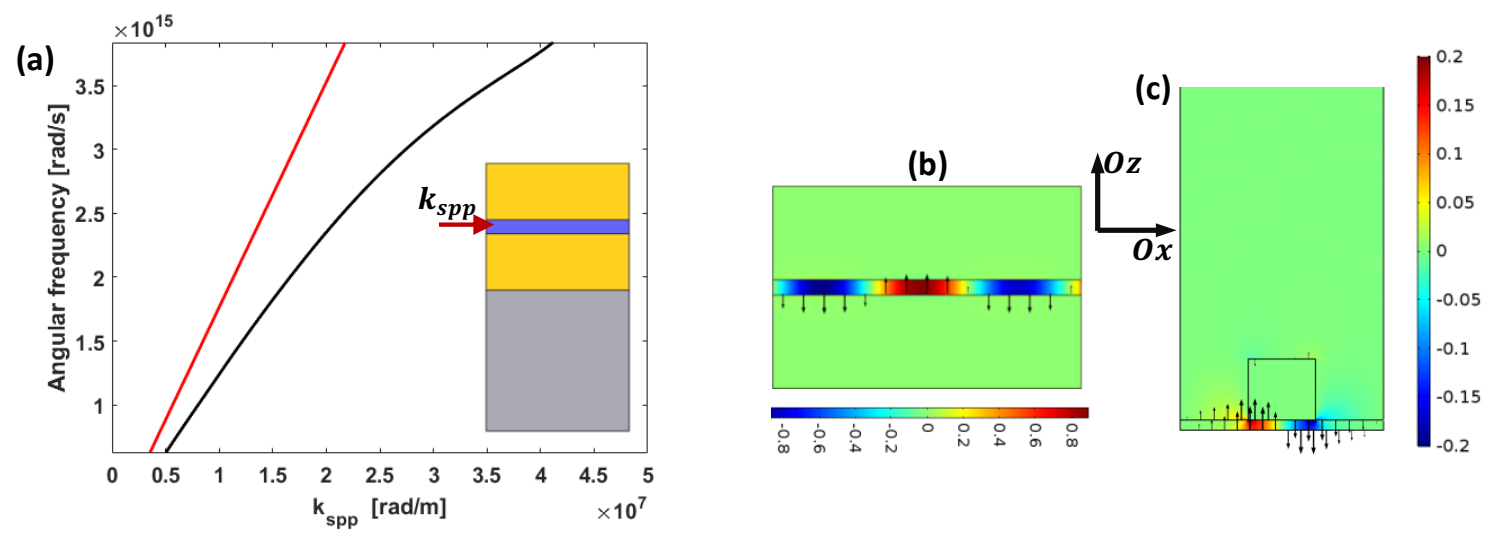

Fig. 3. (a) Dispersion curve in black line of a 2D MIM-waveguide system. The latter structure consists in silicon substrate (infinite)/ $\mathrm{Au}(300 \mathrm{~nm}) /$ polyamide polymer $(50 \mathrm{~nm}) / \mathrm{Au}(300 \mathrm{~nm})$. Light line in polyamide polymer material is plotted in red along with the dispersion curve. A schematic of the structure is shown in the inset. (b) Transverse component $E_{Z}$ of the electric field map of propagative surface plasmon in the system of (a); black arrows indicating the $E_{Z}$-lines are also shown. (c) Same as in (b) but for the MIM-LSP confined in the MIM-cavity of the structure of Fig. 2(a).

It is worthwhile noting that $E_{z}$-field arrows (Fig. 3(c)) indicate that $E_{z}$ reaches its maximum near the MIM-cavity edges; on the other hand $E_{z}$ can be expressed as:

$$
E_{z}(\rho, \phi)=\cos (m \phi) J_{n}\left(k_{s p p} \rho\right)
$$


where, $J_{n}$ is the Bessel function of first kind of order $n(n \in \mathbb{N}),(\rho, \phi)$ are the polar coordinates and $m \in \mathbb{N}$. Hence, the plasmonic standing waves confined in the MIM-cavity should approximately verify the condition:

$$
\frac{\partial E_{z}}{\partial \rho}(\rho=R)=0 \Rightarrow J^{\prime}{ }_{n}\left(k_{s p p} R\right)=0
$$

If we denote by $x_{m n}$ the zeros of Bessel function's derivative, then the dispersion relation of the plasmonic standing waves within the MIM-cavity is:

$$
k_{s p p}(d, \lambda)=\frac{x_{m n}}{R}
$$

Based on Fig. 3(c), the confined MIM-LSP mode in the MIM-cavity correspond to the first zero of $J^{\prime}{ }_{n}\left(k_{s p p} R\right)$ i.e., $x_{11} \approx 1.841$, which means that $m=1$ and $n=1$ for this mode. It is worthy to mention that although the MIM-LSP mode observed in Fig. 2 is essentially confined at the bottom of AuNCs, it still couples with localized plasmons of the neighbouring AuNCs. As a matter of fact, we have noticed that the absorbance spectrum of the system (of Fig. 2(a)) is fairly sensitive to lattice parameter $a$. Corresponding result is presented in S.I. (Fig. S2) where $a$ is varied from $1000 \mathrm{~nm}$ up to $1400 \mathrm{~nm}$ (remaining parameters being unchanged). Increasing of $a$ results in almost linear redshift of the plasmonic mode frequency until $a \geq 1500 \mathrm{~nm}$ where the redshift saturates.

\section{Plasmon-phonon coupling in the periodic structure}

In this section, we study the effect of localized and Sezawa elastic modes (3)-(8) on the plasmonic properties of the structure of Fig. 1(a). To that regard, we use the so-called moving interface (or moving mesh) effect which accounts for the acoustic induced deformations along each interface (between two materials) of the system [12]. Due to the fact that an acoustic period $\sim 10^{8} / \mathrm{s}$ is several orders of magnitude smaller than an optical cycle of the order of $10^{14} / \mathrm{s}$, we use the quasi-static approximation in which at an instant of time of the deformed geometry we calculate the new resonance plasmon mode frequency. Specifically, we simulate the absorbance spectrum or the optical eigenfrequency of the structure for different phases $\varphi=2 \pi f t$ during an acoustic period, where $f$ is the eigen-frequency of the elastic mode of interest and $t$ is time. The deformation amplitude applied via the moving mesh effect is assumed to be such that the maximum elastic displacement inside the nanocylinder equals to $1 \%$ of its height, which yields $u_{\max }=3 \mathrm{~nm}$. This value is relatively high as compared to those produced by an acoustic surface wave generated by interdigital transducers in front of the structure. Nonetheless, owing to the linear dependence of the plasmon eigenfrequency modulations with the amplitude of the acoustic deformation, we use such higher values for the sake of the numerical simulations which can be then scaled to smaller deformations. In order to assess the plasmon-phonon coupling magnitude, we will investigate the evolution of the plasmon eigen-wavelength versus the elastic phonon phase $\varphi$. Specifically, we compute the absorption coefficient for three different values of $\varphi$ to illustrate how the former can be shifted, and on the other side we simulate the plasmon eigen-wavelength for a set of values $\in[0-\pi]$.

\section{(a)}

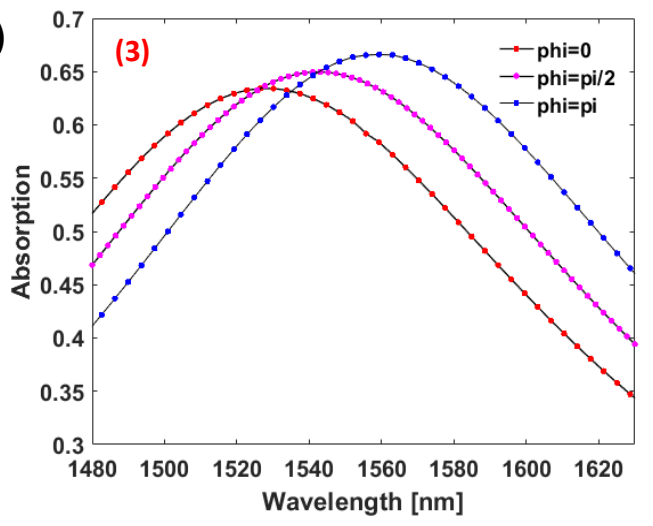

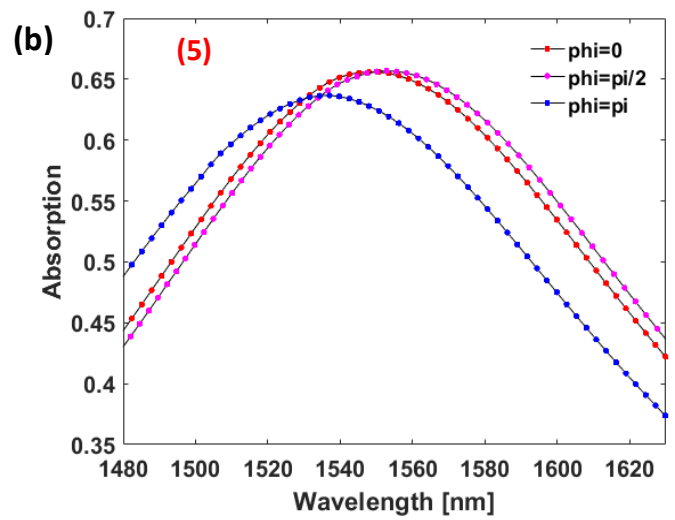



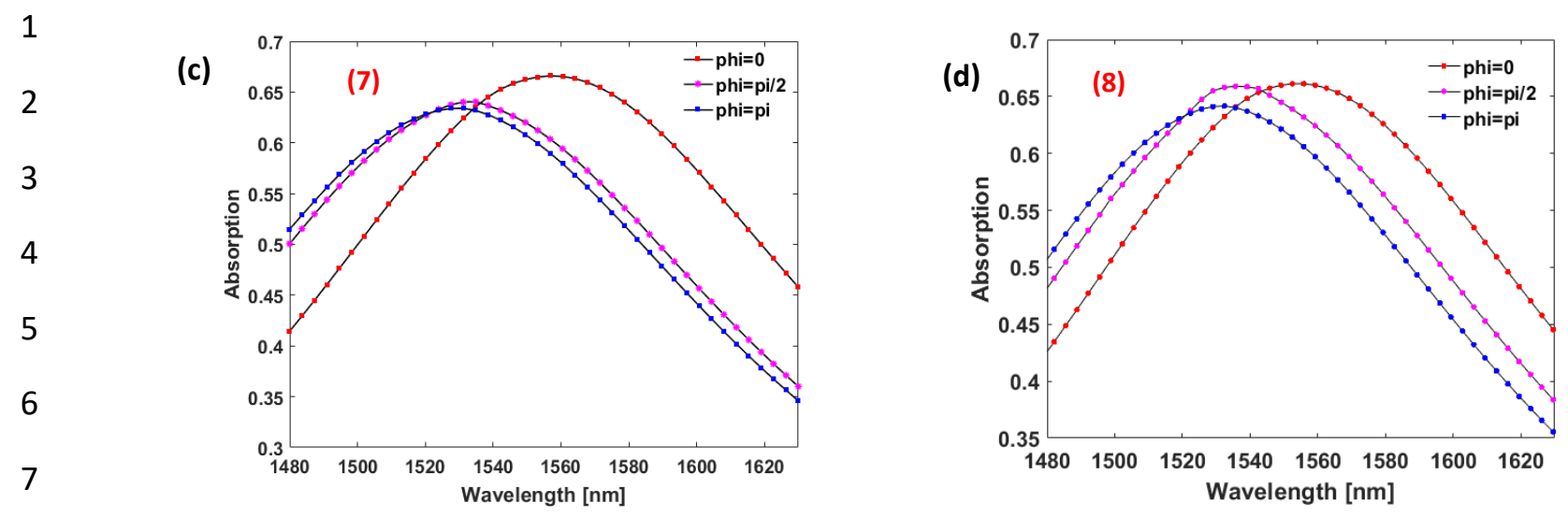

\section{(e)}
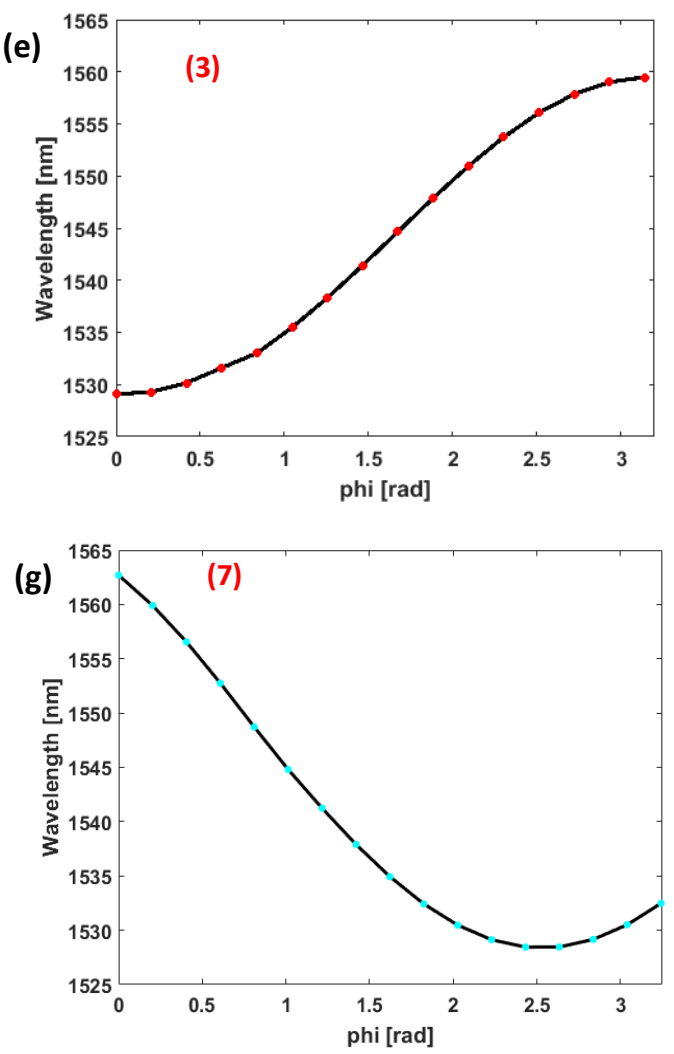

(f)
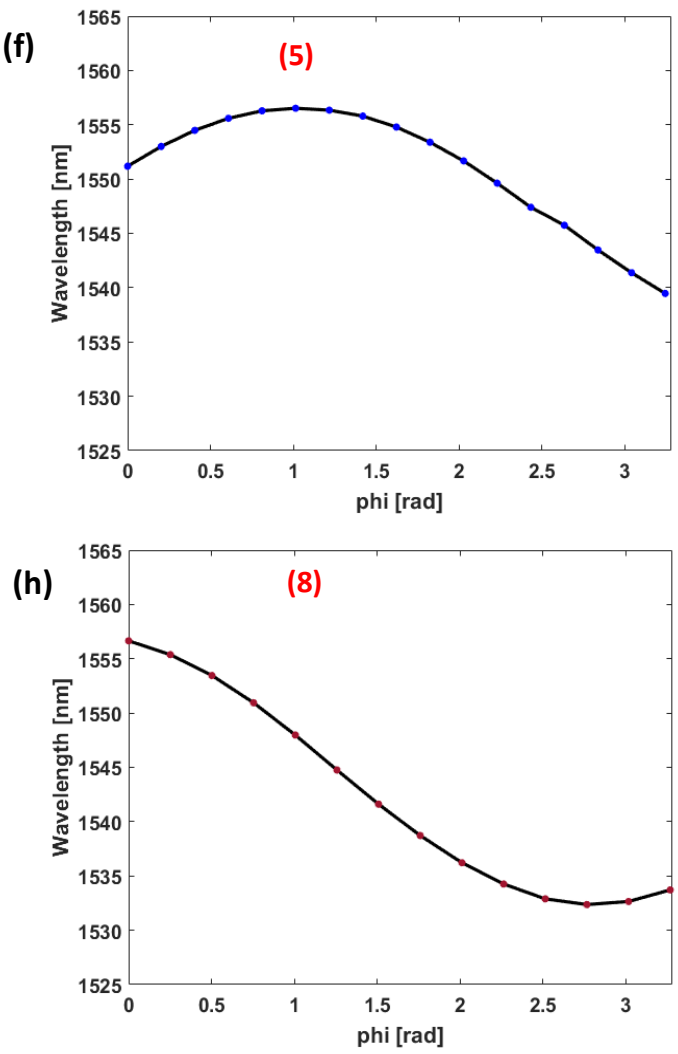

Fig. 4. (a)-(d) Absorbance spectra of the system depicted in Fig. 1(a) for three different values of the phase $\varphi$ of localized elastic mode (3) and Sezawa modes (5), (7) and (8) of Fig. 1, to which we refer by their numbers in each panel. (e)-(h) Simulated eigenwavelength (in vacuum) of the plasmon mode of the MIM-cavity as a function of the phase $\varphi$ for modes (3), (5), (7) and (8), respectively.

In Figs. 4(a)-4(d), we present the absorbance spectra of the system for the values of the phase $\varphi=0, \pi / 2, \pi$ for the elastic modes (3), (5), (7) and (8), while in S.I. we give the absorbance spectra for the modes (4) and (6) that display a weak phonon-plasmon coupling (Figs. S3(a)-S3(b)). Clearly, for modes (3), (5), (7) and (8) the absorption coefficient undergoes an important shift as its maximum position changes with $\varphi$. Conversely, modes (4) and (6) exhibit almost no effect (see Figs. S3(a)-S3(b)) except a very little change in the absorption spectrum shape for mode (4) between the case $\varphi=0$ and $\varphi=\pi / 2, \pi$, which we attribute to the mesh refinement degree in numerical 
as a function of $\varphi$ for modes (3), (5), (7) and (8). As for modes (4) and (6) the results are presented in Figs. S3(c)S3(d). It is worth pointing out that the simulated eigen-wavelength corresponds approximately to the wavelength of the maximum in the absorption spectrum. One notes that localized elastic mode (3) and Sezawa mode (7) induce the strongest plasmon-phonon coupling magnitudes as the plasmon mode frequency maximum modulation amplitude reaches a value of $\Delta \lambda_{\text {max }} \approx 31 \mathrm{~nm}$ between $\varphi=0$ and $\varphi=\pi$ for mode (3) and $\Delta \lambda_{\max } \approx 34 \mathrm{~nm}$ for mode (7) (Figs. $4(\mathrm{e})$ and $4(\mathrm{~g})$, resp.). On the other hand, the modulation amplitudes for modes (5) and (8) amount to $\Delta \lambda_{\max } \approx 17 \mathrm{~nm}$ (Fig. 4(f)) and $\approx 25 \mathrm{~nm}$ (Fig. 4(h)), respectively. The observed modulation amplitudes characterized by $\Delta \lambda_{\max }$ measure the phonon-plasmon coupling strength and, we can notice that overall they are consistent with the predictions made in section 2 based on the analysis of the shapes of modes (3), (5), (7) and (8) and how they would deform the effective MIM-cavity. Concerning modes (4) and (6) the value of $\Delta \lambda_{\max }$ tends to zero (see Figs. S3(c)S3(d)) in agreement with the behavior of absorbance versus $\varphi$, observed in Figs. S3(a)-S3(b).

\section{Plasmon-phonon coupling based on the generation of surface acoustic waves}

\subsection{Transmission based on SAW}

In this section, we investigate the coupling of localized and Sezawa elastic modes of the structure with the studied plasmon mode of the MIM-cavity using the generation of surface acoustic waves in front of the plasmonic structure. This is to provide proof of concept by showing numerically how the plasmon-phonon coupling with the proposed geometry design could be experimentally realized. As a first step, we will focus on the analysis of the transmission properties of the system based on surface elastic waves. Figure 5(a) shows a sketch of the geometry design of the studied structure. For simplicity, we chose to set one infinitely periodic row of gold pillars roughly in the middle of the structure on top, along $y$-axis; namely there only one unit cell along $x$-axis. The system is finite along the propagation direction $(O x)$. Perfect matched layers are placed all around the structure in order to get rid of undesired reflections perturbing the system. The geometrical parameters characterizing the structure such as the gold nanocylinder height and radius, the period of the grating (along $O y$ axis) and the materials layers thicknesses are kept unchanged as previously. To excite the system we employ a vertical force along a line source on top of the polymer material as shown in Fig. 5(a). In Fig. 5(b), we show the displacement field $\sqrt{|u|^{2}+|v|^{2}+|w|^{2}}$ map within the structure for an incident plane wave at the frequency $f=510 \mathrm{MHz}$ (this almost matches the frequency of the cylinder compression mode studied above). Fig. 5(c), depicts the $w$-component of the field within the median cutplane $(O z x)$ of the system. Based on these figures, we observe the strong confinement of the propagating waves close to the multilayer surface, indicating the excitation of a Sezawa surface wave. In order to study the interaction of the incident surface wave with the row of AuNCs we have simulated the transmission spectrum of the structure (of Fig. 5(a)); the frequency span extends from $400 \mathrm{MHz}$ up to $800 \mathrm{MHz}$ which ensures to include compressional mode (3) and degenerate flexural mode (4) of the cylinder. Figure 5(d) gives the corresponding results, where we note the excitation of two localized modes since two dips show up in the transmission spectrum. The dips are situated at $f_{1} \approx$ $513 \mathrm{MHz}$ and $f_{2} \approx 584 \mathrm{MHz}$ which can respectively be associated with the compressional mode (3) of the AuNCs at $508 \mathrm{M} \mathrm{Hz}$ and flexural mode (4) at $580 \mathrm{M} \mathrm{Hz}$ in Fig. 1(a).

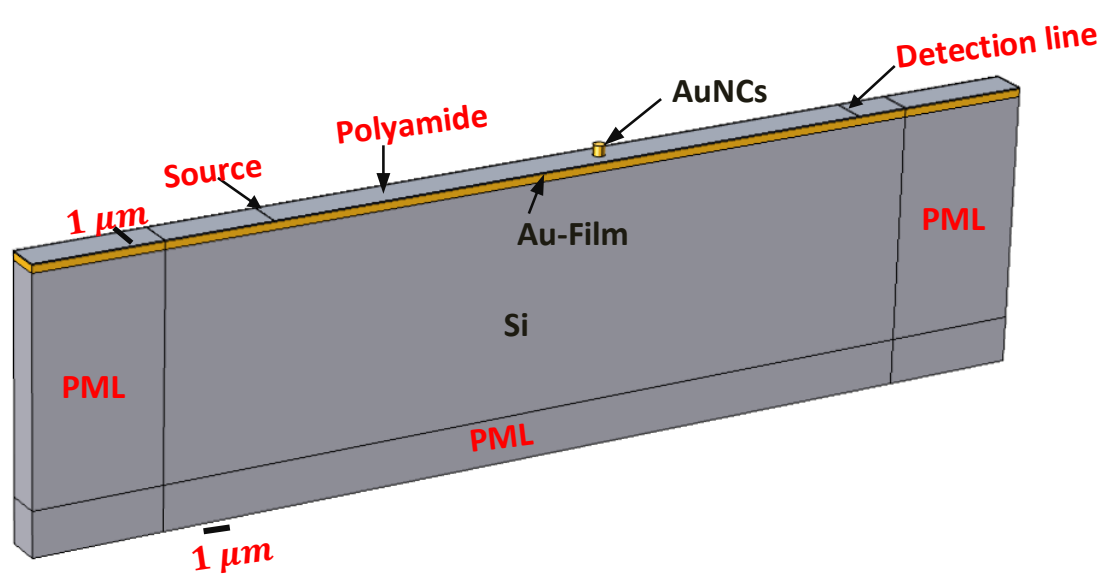


(b)

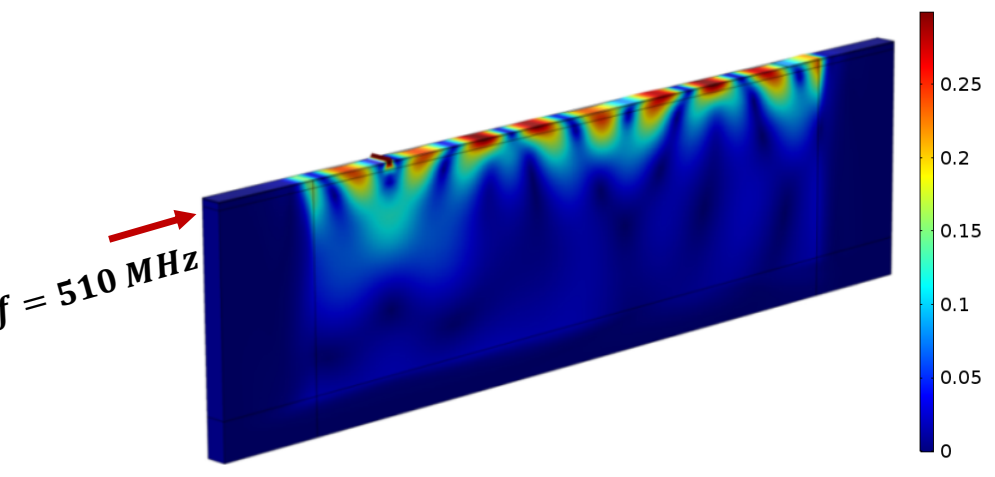

(c)

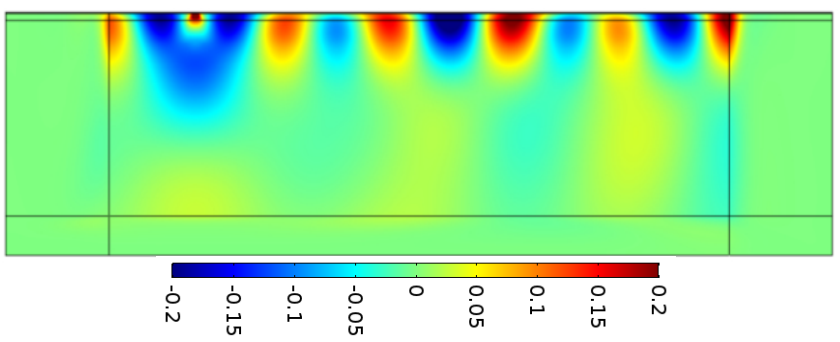

(d)

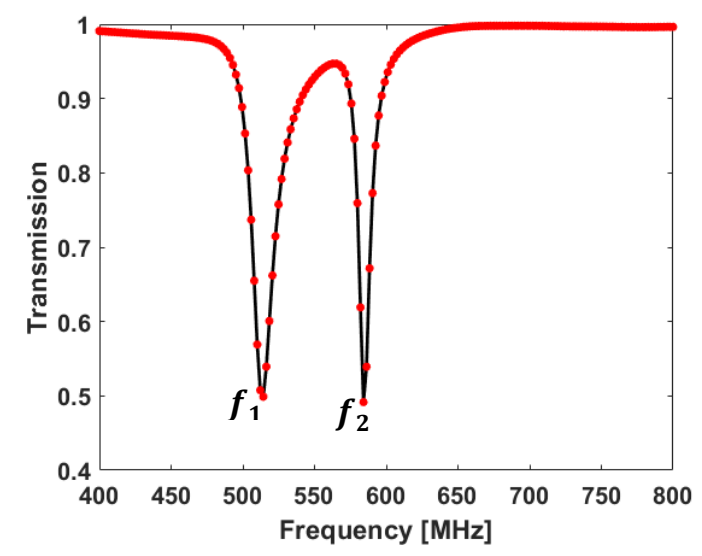

(e)

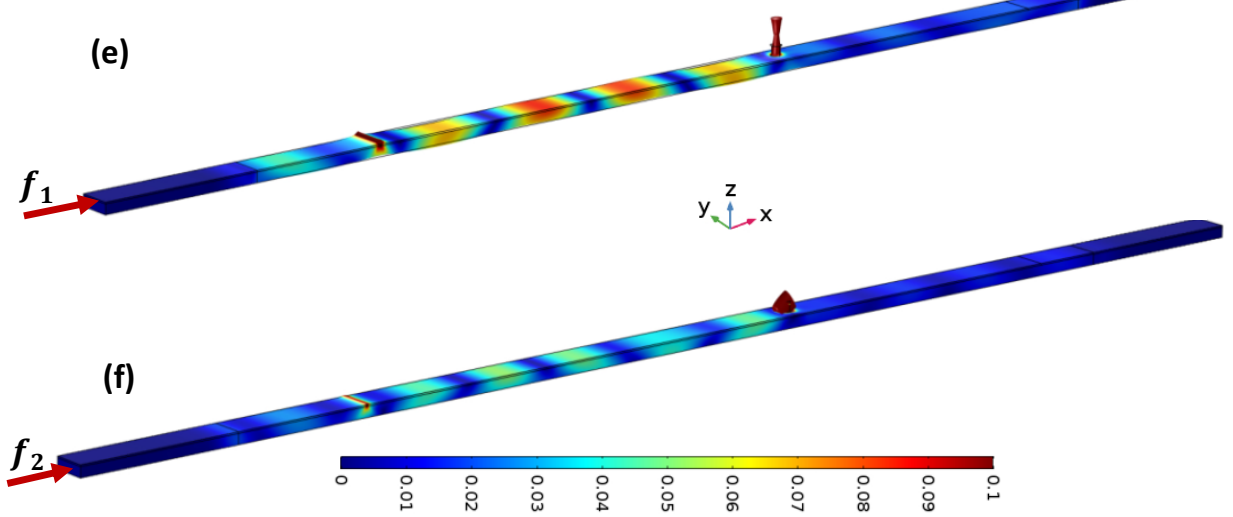

Fig. 5. (a) Schematic of the studied geometry design used for the transmission of surface elastic waves. It consists in one periodic row of AuNCs along $y$-axis, deposited on multilayer silicon substrate as in Fig. 1(a)) and surrounded with PML domains, so to get rid of undesired reflections. (b) Displacement field norm map within the structure associated with a propagating Sezawa surface wave at the frequency $f=510 \mathrm{MHz}$. (c) Map of the $w$-component of the displacement field in $O x z-$ cut plane of the system (median plane). (d) Simulated transmission spectrum of the structure depicted in (a). (e)-(f) Displacement field (norm) map at the frequencies of the observed dips in (d), that is, at $f_{1} \approx 513 \mathrm{MHz}$ and $f_{2} \approx 584 \mathrm{MHz}$, respectively. 
This is confirmed in Figs. 5(e)-5(f), where we present the displacement field map within the upper layers (including PMLs) of the system for clarity, at the dips frequencies. It can be seen that the incident surface waves excite and strongly couple with compressional (Fig. 5(e)) and flexural modes (Fig. 5(f)) of the nanocylinder, resulting in the reflection of waves towards the system inlet.

5

\subsection{Localized surface plasmon of the system}

In this part, we briefly look at the MIM-cavity plasmon modes displayed by the system depicted in Fig. 5(a) at around $\sim 1543 \mathrm{~nm}$. The latter is different from the periodic structure of Fig. 1(a) as it is constituted by only one unit cell along $(O x)$ instead of a periodic array. Subsequently, an adapted geometry for optical simulations should be set in order to determine the plasmonic modes of the system. Figure 6(a) presents such geometry where an air medium is added on top of AuNCs/Si-substrate and PML media removed as we aim to perform eigen-frequency search (for plasmonic modes). Numerical details concerning the optical simulations of the system are given in supplementary information. In Figs. 6(b) and 6(c), we give the electric field norm map within the simulated mediums of the structure, where it can be noticed that excited plasmonic mode corresponds well to the MIM-cavity plasmon mode studied in section 3 above. This is particularly explicit when comparing the mode structure observed here (Fig. 6(c)) in $o x y$-cut plane with the one seen in Fig. 2(e). The eigen-wavelength (in vacuum) of the MIM-cavity plasmon mode observed here is about $\lambda_{s p p} \approx 1602 \mathrm{~nm}$, which is redshifted comparing with the value obtained above $\left(\lambda_{s p p} \approx\right.$ $1543 \mathrm{~nm})$.

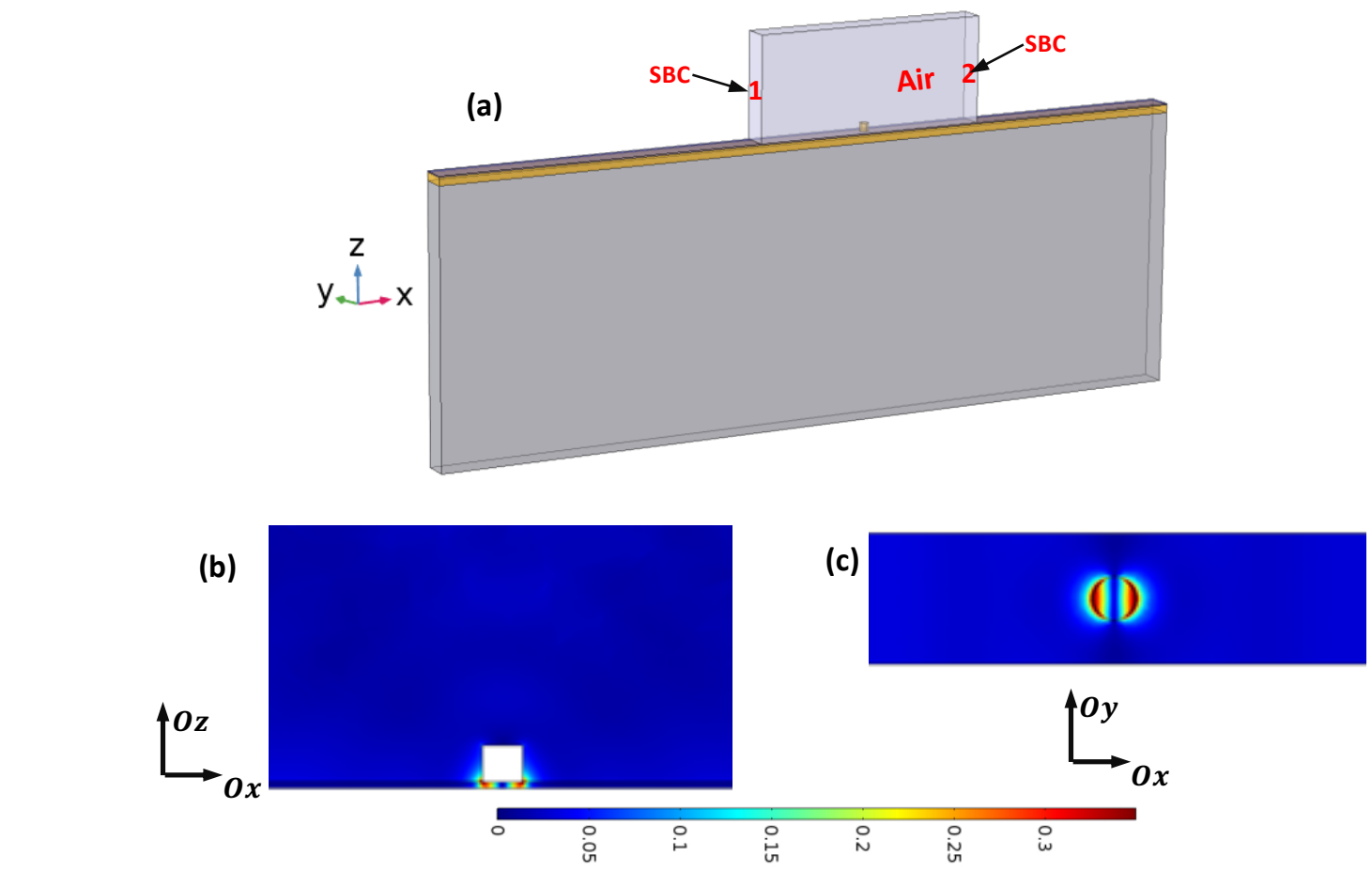

Fig. 6 (a) Sketch of the geometry platform used for phonon-plasmon coupling using incident surface elastic waves as a mean for the excitation of localized phonons in the gold pillars. The structure is similar to the one in Fig. 5(a) except that an air medium is added on top of the gold nanocylinders for optical simulations. PMLs surrounding the system are not shown for simplicity. At facets 1 and 2 delimiting the air domain, scattering boundary conditions (SBC) are applied to prevent undesired reflections at these boundaries. (b)-(c) Electric field norm map within 2D-cut planes of the system (ozx) and (oxy), respectively in the parts of the structure where optical simulations are performed.

This can be explained based on Fig. S2, where we have noticed that increase of lattice parameter $a$ or equivalently the distance between AuNCs redshifts the plasmon mode frequency (revealing the role of interactions between AuNCs in their optical response). Indeed, in the studied system here there is one periodic row of AuNCs on top along $y$-axis which is similar to the case where $a$ is increased (to infinity along $x$-axis) in the periodic structure of Fig. 1(a). The calculations are realized as previously at different phases $\varphi \in[0-\pi]$ of the acoustic surface wave. 
As concerning the applied deformation, we set the value of the linear force exciting the incident surface wave so that maximum elastic displacement within gold pillars is of the order of $u_{\max } \approx 3 \mathrm{~nm}$, which is equivalent to maximum displacement set in AuNCs in the infinitely periodic system studied in section 4 above. Specifically, the application of a linear force of $F \approx 22 \mathrm{~N} / \mathrm{m}$ yields a maximum displacement in gold pillars equals to $u_{\max } \approx 2.98 \mathrm{~nm}$. Simulation result is reported in Fig. 7(a), where we can observe that plasmonic mode wavelength gets modulated by excited localized phonon via incident surface wave, with maximal oscillation amplitude of about $\Delta \lambda_{\text {sppmax }} \approx$ $19.9 \mathrm{~nm}$. The latter value is nearly $36 \%$ smaller than the one observed in Fig. 4(e). This relates to sensitivity of phonon-plasmon coupling magnitude to lattice parameter value $a$ (or distance between Au-pillars), reflecting the influence of interactions between Au-pillars on phonon-plasmon coupling strength. Indeed, we notice in Fig. 7(b) where we have plotted $\Delta \lambda_{\text {sppmax }}$ (for mode (3)-plasmon coupling) versus lattice parameter $a$ in periodic system of Fig. 1(a) that increase of $a$ from $1000 \mathrm{~nm}$ up to $1400 \mathrm{~nm}$ results in drop of $\Delta \lambda_{\text {sppmax }}$ of about $\approx 27 \%$, which is close to $\Delta \lambda_{\text {sppmax }}$ drop seen in Fig. 7(a) (where depicted results correspond to the system of Fig. 5(a) for which $a$ is infinity).

The excitation of the Sezawa surface modes (5) and (7) for the purpose of their coupling with plasmonic mode is different due to their dispersion; indeed at a certain frequency one specific associated wave vector $k_{x}$ should be set for each mode. For instance, if we consider modes (5) and (7) at the frequency $f=0.8 \mathrm{GHz}$, then associated wave vectors should be respectively $k_{x} \approx 2.1877 .10^{6} \mathrm{rad} / \mathrm{m}$ and $k_{x}=1.4305 .10^{6} \mathrm{rad} / \mathrm{m}$, based on the system dispersion curve shown in red in Fig. 1(c). Numerically, the setting of the incident surface wave $k_{x}$-value is realized by placing six linear sources (of vertical force) parallel to each other on top of the structure inlet; the sources should all be set apart from each other by an amount exactly equal to $a_{x}=2 \pi / k_{x}$. In Fig. 7(c), we depict the displacement field norm map $\sqrt{|u|^{2}+|v|^{2}+|w|^{2}}$ within the structure associated with excited Sezawa mode at $f=$ $0.8 \mathrm{GHz}$ and $k_{x}=2.1877 .10^{6} \mathrm{rad} / \mathrm{m}$.

\section{(a)}

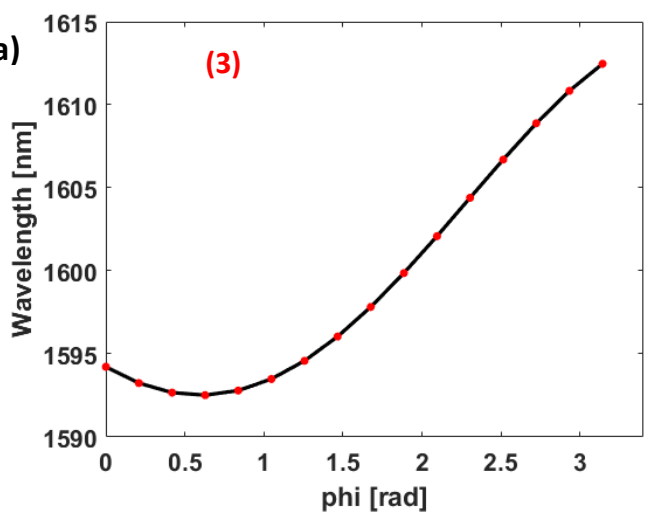

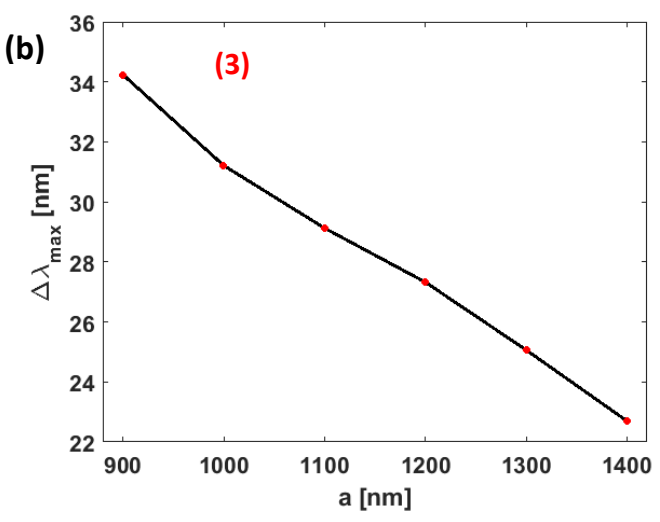

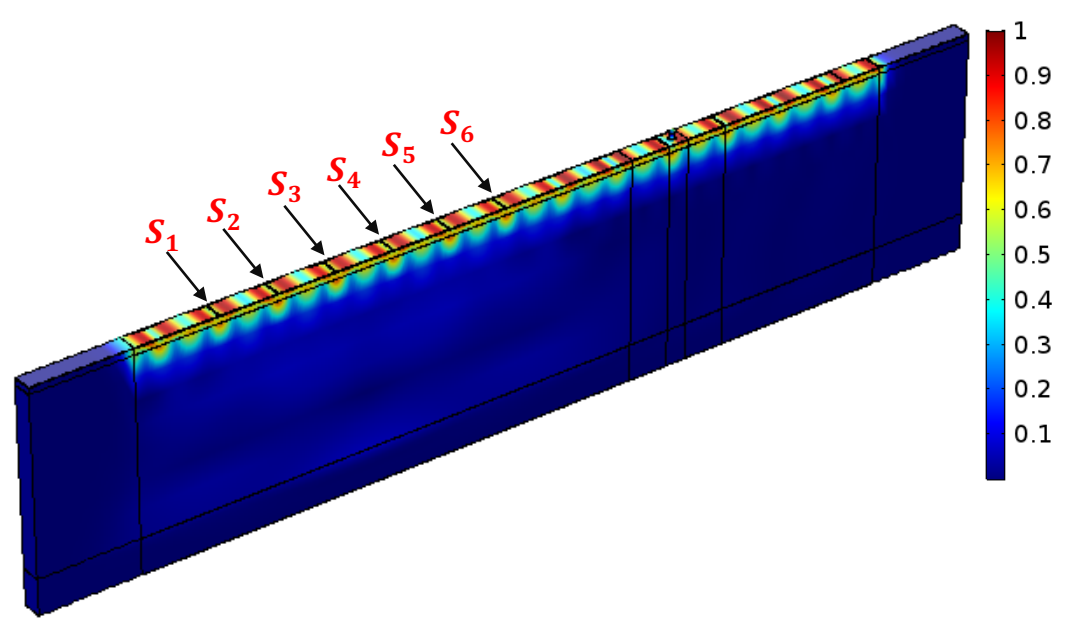


(d)

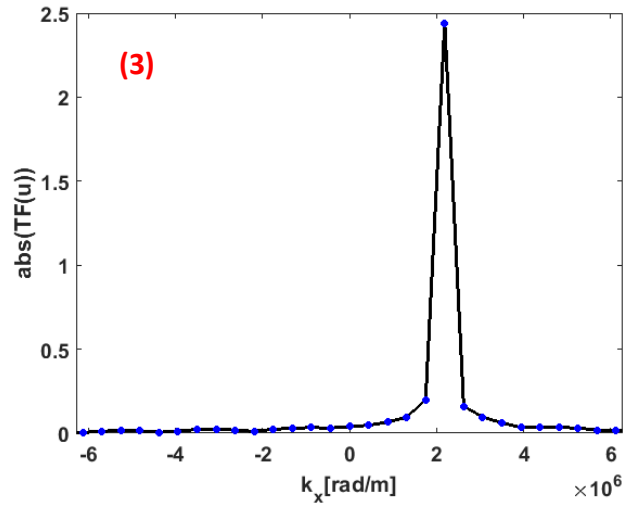

(f)

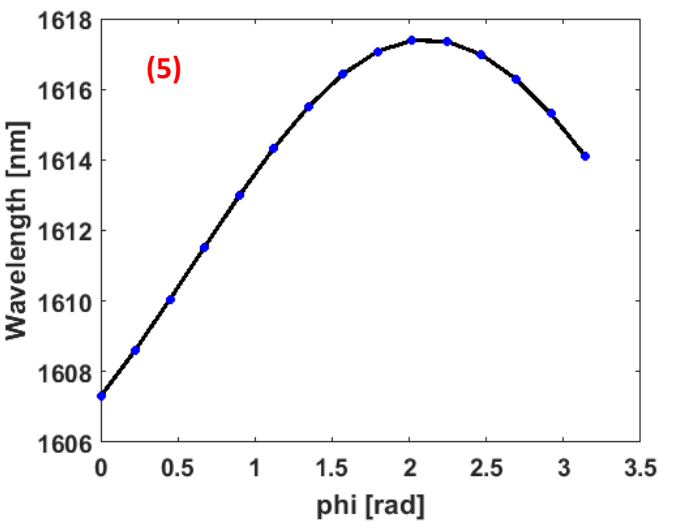

(e)
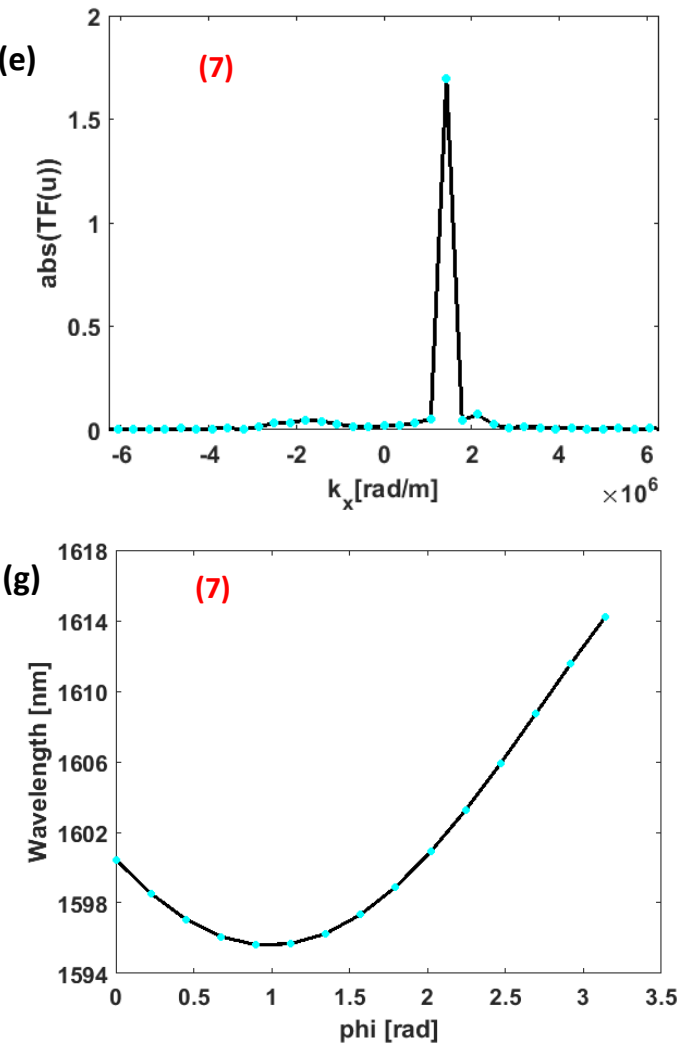

Fig. 7. (a) Plasmonic mode eigen-wavelength (in vacuum) as a function of incident elastic surface wave phase $\varphi \in[0-p i]$. Frequency of the latter is set at $f=510 \mathrm{MHz}$, so to match localized mode (3) frequency. As a result, the latter mode is excited by incident SAW and couples with the plasmon. (b) Maximum amplitude oscillation of the plasmon eigen-wavelength $\Delta \lambda_{\text {sppmax }}$ as modulated by localized elastic mode (3) versus lattice parameter $a$ in case of periodic multilayer system depicted in Fig. 1(a). (c) Displacement field norm map associated with Sezawa mode (5) (seen in Fig. 1(h)) within the system depicted in Fig. 5(a). The mode is excited by placing six linear sources of force parallel to each other on top of the structure inlet (on top of polymer material); the sources are distant by an amount equals to $a_{x}=2 \pi / k_{x}$ such that $k_{x}=2.1877 .10^{6} \mathrm{rad} / \mathrm{m}$. Associated frequency value set is $f=0.8 \mathrm{GHz}$ (based on Fig. 1(c)). (d) Fourier transform performed along $(O x)$ of $x$-component of displacement field (u) associated with Sezawa modes (5) depicted in (b). (e) Same as (c) but for Sezawa mode (7). (f)-(g) Plasmon mode eigenwavelength plotted against acoustic phase $\varphi \in[0-p i]$ of Sezawa modes (5) and (7), respectively. Numbers in each figure refer to the studied elastic modes.

The linear sources are highlighted by $S_{i}$, where $i$ is such as $1 \leq i \leq 6$. In Fig. S4(c), we perform a zoom-in on the part of the structure surrounding the AuNCs in order to compare with the mode structure shown in Fig. 1(h) (corresponding to the periodic system of Fig. 1(c)). Furthermore, in Fig. 7(d) we show the spatial Fourier transform, performed along the $x$-axis, of the $x$-component $(u)$ of the excited mode displacement field, so to check out if the associated $k_{x}$-wave vector corresponds well to the correct value set (i.e., $k_{x}=2.1877 .10^{6} \mathrm{rad} / \mathrm{m}$ ). One notes that the mode structure in Fig. 6(c) is very nearly the same as in Fig. 1(h), and that Fourier transform of its $u$-component is rightly centered at $k_{x} \approx 2.189 .10^{6} \mathrm{rad} / \mathrm{m}$, indicating successful excitation of Sezawa mode (5). Similarly, we give in Fig. S4(d) and in Fig. 7(e) respectively a zoom-in around AuNCs of displacement field map within the system associated with Sezawa mode $(7)\left(f=0.8 \mathrm{GHz}\right.$ and $\left.k_{x}=1.4305 .10^{6} \mathrm{rad} / \mathrm{m}\right)$ and the spatial Fourier transform of its $u$-component. Here again, the observed mode structure is patently quasi-identical to the one sketched in Fig. $1(\mathrm{j})$, and Fourier transform peaks around $k_{x} \approx 1.4327 .10^{6} \mathrm{rad} / \mathrm{m}$ which matches the right wave vector value for mode (7) at $f=0.8 \mathrm{GHz}$.

Displacement field $(u, v, w)$ associated with Sezawa modes (5) and (7) is used to deform the mesh, so to study phonon-plasmon coupling. Maximum elastic displacement field norm near or within gold pillars associated with studied modes is set to be of the order $\approx 3 \mathrm{~nm}$ as previously by adjusting the strength of the force applied to the line sources. In the aim of comparing phonon-plasmon coupling strength between Sezawa and localized compressional 
modes, it is important that maximum displacement near or in AuNCs associated with the modes is being equivalent. In that spirit, we set linear force value for Sezawa modes (5) and (7) to $F \approx 110 \mathrm{~N} / \mathrm{m}$ and $F \approx 62 \mathrm{~N} / \mathrm{m}$ resp., which yields a maximum displacement near AuNCs of about $u_{\max } \approx 3 \mathrm{~nm}$ for both modes. From this result, it is also worthwhile noticing that for a given strength of the forces applied to line sources, the efficiency of exciting different Sezawa modes is not the same.

In Figs. 7(f) and 7(g), we report corresponding simulation results where plasmon mode eigen-wavelength value is plotted against Sezawa modes acoustic phase $\varphi \in[0-\pi]$. It can be seen that plasmonic mode eigen-wavelength is effectively modulated by Sezawa modes such as the maximum amplitude of oscillation is about $\Delta \lambda_{\text {sppmax }} \approx 10 \mathrm{~nm}$ for mode (5) and $\Delta \lambda_{\text {sppmax }} \approx 19 \mathrm{~nm}$ for mode (7). As noticed above concerning the localized mode (3), the values of $\Delta \lambda_{\text {sppmax }}$ seen here are smaller compared with the values taken by this parameter in Figs. 4(f) and $4(\mathrm{~g})$. This is related to the same reason mentioned earlier, that is, the structure only contains one unit-cell along $x-$ axis $(a \rightarrow \infty)$ which results in a decrease of $\Delta \lambda_{\text {sppmax }}$. In fact, we depict respectively in Figs. S4(a) and $\mathrm{S} 4$ (b) $\Delta \lambda_{\text {sppmax }}$ as a function of $a$ for modes (5) and (7) in the periodic system of Fig. 1(a), where one notes decrease of $\Delta \lambda_{\text {sppmax }}$ as $a$ increases. Specifically, increasing of a from $1000 \mathrm{~nm}$ to $1400 \mathrm{~nm}$ results in $\Delta \lambda_{\text {sppmax }}$ drop of roughly $29 \%$ for mode (5) and $36.4 \%$ for mode (7), while the drop of $\Delta \lambda_{\text {sppmax }}$ in Figs. $7(\mathrm{f})$ and $7(\mathrm{~g})$ is of about $41.2 \%$ and $44 \%$ for modes (5) and (7), respectively. The latter percentage drops are slightly bigger than the formers, but they correspond to the system of Fig. 5(a) for which $a$ is infinity indicating that the trend observed in Fig. S5(a)-S5(b) is quite coherent with these numbers. Comparison of the value of $\Delta \lambda_{\text {sppmax }}$ between different studied modes yields the following: $\Delta \lambda_{\text {sppmax }}(\operatorname{mode}(3)) \approx 19.9 \mathrm{~nm} \succsim \Delta \lambda_{\text {sppmax }}(\operatorname{mode}(7)) \approx 19 \mathrm{~nm}>\Delta \lambda_{\text {sppmax }}(\operatorname{mode}(5)) \approx 10 \mathrm{~nm}$, which matches roughly the same trend as observed above in Fig. 4.

To sum up, these results indicate that for incident surface waves with amplitude of just few nanometers $(\sim 3 \mathrm{~nm})$ that strongly overlap with the plasmon (Sezawa modes 7 and 5) or for those leading to the excitation of localized phonons such as mode (3) (with a similar amplitude), strong phonon-plasmon coupling arises based on the observed important plasmon frequency modulation $(\sim 19 \mathrm{~nm})$. It is important to highlight that comparable results have been reported in [29], where strong phonon-plasmon coupling is achieved with incident surface waves having amplitudes of $\sim 4 \mathrm{~nm}$ and producing a plasmon frequency modulation up to $\sim 14 \mathrm{~nm}$. It is worth mentioning that recently ultrathin metal films have been shown to manifest new dispersion regimes [55], and a strong tunability of plasmonic resonances supported by these films by hundreds of nanometers as one of their dimensions is changed by hundreds of nanometers also. In terms of comparison, the plasmon in our work (Fig. S2) is tunable with $\approx 25 \mathrm{~nm}$ as the lattice parameter is adjusted by hundreds of nanometers, showing less but in the same order of magnitude sensibility as compared to the work in [55]. As mentioned earlier, a tuning of the plasmon frequency of $\sim 19 \mathrm{~nm}$ is attained in our system due to incident SAWs, which is also smaller but in the same range scale of the values reported in [55] taking into account the small amplitude $(\sim 3 \mathrm{~nm})$ of incoming SAWs. Overall, the proposed SAW-based platform enables the reach of strong optomechanical interactions, paving the way of various possible applications such as ultrasensitive SAW-sensors.

\section{Conclusion}

3D finite element based-numerical simulations have been carried out in this paper to study surface acoustic waves interaction with localized surface plasmons in a multilayer structure supporting Au pillars. Firstly, we have studied phononic and optical properties of a periodic pillared multilayer system. Four low frequency localized elastic modes within AuNCs are identified and three branches corresponding to Sezawa modes confined near the surface of the system are observed. The latters modes arouse out of the interaction of Sezawa modes localized in Au-thick film and localized modes in AuNCs. Optical simulations reveal a strong resonance at $1543 \mathrm{~nm}$ where absorption coefficient reaches almost $64 \%$; the resonance is shown to correspond to so-called MIM-localized surface plasmon mode. This mode is strongly confined at the bottom of AuNCs. Using moving mesh effect, we have investigated the interaction of localized elastic and Sezawa modes with the plasmonic mode by simulating eigen-frequency (or absorbance) of the plasmon versus the phase of acoustic modes. It is found that localized compression and Sezawa modes belonging to upper (unfolded) branch induce the higher coupling magnitude, followed by lower branch Sezawa modes. This is related to these modes localization which is such that they compress/stretch the MIM-like cavity volume underneath AuNCs while oscillating. Surface acoustic waves interaction with the plasmon is performed in a finite multilayered 
system having one periodic row of AuNCs deposited on its top. SAW are excited at the structure inlet in front of gold pillars row and their coupling with the latters results in the excitation of localized phonons in AuNCs. We show using moving mesh effect that localized plasmon eigen-frequency is effectively modulated as a function of SAW temporal phase. We uncover the role of interactions between the pillars on the phonon-plasmon coupling magnitude by showing how the latter drops as the distance between the formers increases. As in the periodic multilayer system, compression and Sezawa modes of the upper (unfolded) branch induce the higher coupling rate followed by the lower branch Sezawa mode. Localized and Sezawa modes that strongly couple with the plasmon might be quite useful for an experimental realization based on the proposed geometry design which in turn may greatly help the design of novel nano-optomechanical devices. Besides, owing to the sensitivity of its plasmonic response, the proposed platform can be harnessed to develop acousto-optic devices for the detection of high frequency acoustic waves or SAW $(\sim \mathrm{GHz})$. As a matter of fact, our concept is in line with previous works such as in [56] where surface plasmons-polariton sensitivity to immediate dielectric environment has been exploited to sense acoustic waves or in [57] where broadband detection of ultrasound was used for photoacoustic microscopy. Recently, Balram et al [58] used optomechanical-based systems to conceive chip-scale acousto-optic modulation devices. On the other hand, the MIM-cavity in our system where phonons strongly overlap with localized plasmons can be regarded as an effective optomechanical cavity whereby acousto-optic modulation monitored by SAW can be achieved. Furthermore, based on such cavity various potential applications involving enhanced sound-light interaction and fast coherent acoustic control of optomechanical devices can be realized [29]. In addition, the use of pump-probe ultrafast spectroscopy [59] in order to follow-up the dynamic of peak energy shift of the localized plasmon in the MIM-cavity of our system could lead to the detection of ultra-small fast coherent oscillation ( picometers amplitudes) localized in the gold pillar above, and excited by some incident SAWs. Alternatively, the peak energy shift of the localized plasmon in the MIM-cavity versus time may detect picometers amplitudes-wise high frequency incident SAWs (Sezawa modes) as they couple with plasmon. Thus, new nanoscale mechanical ultrasensitive high frequency sensors may be envisaged based on the strong optomechanical effects achieved by our SAW-system as shown in [60]. Acousto-optic devices for light modulation in the IR region specifically are still lacking mainly due to weak phonon-photon interaction efficiency [61]; in that sense the SAW-acousto-plasmonic in our work reaching strong phonon-plasmon coupling around $1.55 \mu \mathrm{m}$ may be employed to design efficient nanoscale acousto-optic light modulators in the infrared region. At last, the proposed SAW-system may be integrated into microfluidic devices whereby SAW driven homogeneous micromixing of multiple laminar flows results in the alteration of the refractive index near the MIMcavity, hence the frequency of the localized plasmon in the latter is modulated, which consequently could lead based on such acousto-plasmofluidic device to the design of modulators, filters, biosensors and optical switches [62].

Supplementary Information (S.I.) contains further simulation data and further details of the numerical method concerning the search for localized plasmons modes of the pillared multilayer structure.

\section{Acknowledgements}

This work was supported by the French National Research Agency ANR as part of the project "RANDOM".

\section{References}

[1] D. A. Fuhrmann, M. T. Susanna, H. kim, D. Bouwmeester, P. M. Petroff, A. Wixforth, H. J. Krenner, Nat Photonics 5, 605-609 (2011).

[2] M. Metcalfe, S. M. Carr, A. Muller, G. S. Solomon, J. Lawall, Phys. Rev. Lett. 105, 037401 (2010).

[3] V. Laude, A. Belkhir, A. F. Alabiad, M. Addouche, S. Benchabane, A. Khelif,and F. I. Baida, Optica 4 (10), 1245-1250 (2017).

[4] S. Völk, F. J. R. Schülein, F. Knall, D. Reuter, A. D. Wieck, T. A. Truong, H. Kim, P. M. Petroff, A. Wixforth, H. J. Krenner, Nano Lett. 10, 3399-3407 (2010).

[5] T. Berstermann, C. Brüggemann, M. Bombeck, A. V. Akimov, D. R. Yakovlev, C. Kruse, D. Hommel, and M. Bayer, Phys. Rev. B 81, 085316 (2010). 
[6] T. J. Kippenberg and K. J. Vahala, Science 321, 1172 (2008).

[7] I. Favero and K. Karrai, Nat Photon 3, 201 (2009).

[8] W. Aspelmeyer, T. J. Kippenberg, and F. Marquardt, Rev. Mod. Phys. 86, 1391 (2014).

[9] M. Aspelmeyer, P. Meystre, and K. Schwab, Physics Today 65, 29 (2012).

[10]E. Gil-Santos, C. Baker, D. T. Nguyen, W. Hease, C. Gomez, A. Lemầtre, S. Ducci, G. Leo, and I. Favero, Nature Nanotechnology 10, 810 (2015).

[11]D. Royer, E. Dieulesaint, Elastic Waves in Solids (Springer, New York, 2000).

[12]A. Yariv, P. Yeh, Optical Waves in Crystals (John Wiley and Sons, New Jersey, 2003).

[13]I. E. Psarobas, N. Papanikolaou, N. Stefanou, B. Djafari-Rouhani, B. Bonello, and V. Laude, Phys. Rev. B 82, 174303 (2010).

[14]M. Maldovan, Nature 503, 209 (2013).

[15]Q. Rolland, M. Oudich, S. El-Jallal, S. Dupont, Y. Pennec, J. Gazalet, J. C. Kastelik, G. Lévêque, and B. Djafari-Rouhani, Appl. Phys. Lett. 101, 061109 (2012).

[16] S. Eljallal, M. Oudich, Y. Pennec, B. Djafari-Rouhani, A. Makhoute, Q. Rolland, S. Dupont, and J. Gazalet, J. Phys.: Condensed Matter 26, 015005 (2014).

[17] M. Eichenfield, J. Chan, R. M. Camacho, K. J. Vahala, and O. Painter, Nature 462, 78 (2009).

[18] M. Eichenfield, J. Chan, A. H. Safavi-Naeini, K. J. Vahala, and O. Painter, Opt. Express 17, 20078 (2009).

[19] J. Chan, A. H. Safavi-Naeini, J. T. Hill, S. Meenehan, and O. Painter, Appl. Phys. Lett. 101, 081115 (2012).

[20] M. Oudich, S. El-Jallal, Y. Pennec, B. Djafari-Rouhani, J. Gomis-Bresco, D. Navarro-Urrios, C. M. Sotomayor Torres, A. Martiınez, and A. Makhoute, Phys. Rev. B 89, 245122 (2014).

[21]J. Gomis-Bresco, D. Navarro-Urrios, M. Oudich, S. El-Jallal, A. Griol, D. Puerto, E. Chavez, Y. Pennec, B. Djafari-Rouhani, F. Alzina, A. Martınez, and C. M. Sotomayor Torres, Nat. Commun. 5, 4452 (2014).

[22]B. Djafari-Rouhani, S. El-Jallal, and Y. Pennec, C. R. Physique 17, 555 (2015).

[23]E. Gavartin, R. Braive, I. Sagnes, O. Arcizet, A. Beveratos, and T. J. Kippenberg, Phys. Rev. Lett. 106,203902 (2011).

[24]D. A. Fuhrmann, S. M. Thon, H. Kim, D. Bouwmeester, P. M. Petroff, A. Wixforth, and H. J. Krenner, Nat. Photon. 5, 605 (2011).

[25]A. H. Safavi-Naeini and O. Painter, Opt. Express 18, 14926 (2010).

[26] M. H. Aram and S. Khorasani, J. Opt. Soc. Am. B 35, 1390-1396 (2018).

[27] J.-C. Hsu, J.-H. Shih, and T.-R. Lin, Appl. Phys. Lett. 111, 021904 (2017).

[28] T.-R. Lin, Y.-C. Huang, and J.-C. Hsu, J. Appl. Phys. 117, 173105 (2015).

[29] T.-R. Lin, C.-H. Lin and J.-C. Hsu, Sci Rep. 5, 13782 (2015).

[30]P. K. Parashar and V. K. Komarala, Sci Rep. 7, 12520 (2017).

[31]E. Palacios, A. Chen, J. Foley, S. K. Gray, U. Welp, D. Rosenmann and V. K. Vlasko-Vlasov, Adv. Optical Mater. 2, 394-399 (2014).

[32] G. Z. Gaydan, O. M. Demkiv, N. Ye. Stasyuk, R. Y. Serkiz, M. D. Lootsik, A. Errachid, M. V. Gonchar and M. Nisnevitch, Appl. Sci 9, 720 (2019).

[33]F. Fathi, M. R. Rashidi, Y. Omidi, Talanta 192, 118-127 (2019).

[34] R. Salahandish, A. Ghaffarinejad, S. M. Naghib, A. Niyazi, K. Majidzadeh-A, M. Janmaleki and A.S.-Nezhad, Sci. Rep. 9, 1226 (2019).

[35] M. Kim, J.-H. Lee and J.-M. Nam, Adv. Sci. 6, 1900471 (2019).

[36] M. R. K. Ali, Y. Wu and M. A. El-Sayed, J. Phys. Chem. C 123, 15375-15393 (2019).

[37] K. Kluczyk, C. David, J. Jacak and W. Jacak, Nanomaterials 9 , 3 (2019).

[38] G. Singh, S. S. Verma, Phys. Lett. A. 383, 1526-1530 (2019).

[39]M. A. van Dijk, M. Lippitz, and M. Orrit, Phys. Rev. Lett. 95, 267406 (2005).

[40]H. Staleva and G. V. Hartland, Adv. Funct. Mater. 18, 3809 (2008).

[41] M. Hu, X. Wang, G. V. Hartland, P. Mulvaney, J. P. Juste, and J. E. Sader, J. Am. Chem. Soc. 125 , 14925 (2003).

[42]N. Large, L. Saviot, J. Margueritat, J. Gonzalo, C. N. Afonso, A. Arbouet, P. Langot, A. Mlayah, and J. Aizpurua, Nano Lett. 9, 3732 (2009).

[43]F. Della Picca, R. Berte, M. Rahmani, P. Albella, J. M. Bujjamer, M. Poblet, E. Cortés, S. A. Maier, and A. V. Bragas, Nano Lett. 16, 1428 (2016).

[44]C. Voisin, N. Del Fatti, D. Christofilos, and F. Vallee, Appl. Surf. Sci. 164, 131 (2000).

[45]A. Nelet, A. Crut, A. Arbouet, N. Del Fatti, F. Vallee, H. Portales, L. Saviot, and E. Duval, Appl. Surf. Sci. 226, 209 (2004). 
[46] G. Bachelier, J. Butet, I. Russier-Antoine, C. Jonin, E. Benichou, and P. F. Brevet, Phys. Rev. B 82, 235403 (2010).

[47]H. Portales, N. Goubet, L. Saviot, S. Adichtchev, D. B. Murray, A. Mermet, E. Duval, and M. P. Pileni, Proc. Natl. Acad. Sci. 105, 14784 (2008).

[48] S. Tripathy, R. Marty, V. Kaixin Lin, S. Lang Teo, E. Ye, A. Arbouet, L. Saviot, C. Girard, M. Yong Han and A. Mlayah, Nano Letters 11, 431 (2011).

[49]A. Mrabti, G. Lévêque, A. Akjouj, Y.Pennec, B. Djafari-Rouhani, R. Nicolas, T. Maurer, P. M. Adam, Phys. Rev. B 94, 075405 (2016).

[50] J. Chan, T. Alegre, A. H. Safavi-Naeini, J. T. Hill, A. Krause, S. Gröblacher, M. Aspelmever and O. Painter, Nature 478, 89 (2011).

[51] M. Soljačić, and J. D. Joannopoulos, Nat. Mater. 3, 211 (2004).

[52] S. Weis, R. Rivière, S. Deléglise, E. Gavartin, O. Arcizet, A. Schliesser, T. J. Kippenberg, Science 330, 15201523 (2010).

[53] L. Bo, C. Xiao, C. Hualin, M. A. Mohammad, T. Xiangguang, T. Luqi, Y. Yi and R. Tianling, J. Semicond. 37, 021001 (2016).

[54]C. Campbell, Surface Acoustic Wave Devices and Their Signal Processing Applications, Publisher: Academic Press Inc, (December 2, 2012).

[55] R. A. Maniyara, D. Rodrigo, R. Yu, J. C.-Ferrer, D. S. Ghosh, R. Yongsunthon, D. E. Baker, A. Rezikyan, F. J. Garcia de Abaio and V. Pruneri, Nat. Photonics 13, 328 (2019).

[56] A. A. Kolomenskii, E. Surovic and H. A. Schuessler, Appl. Opt. 57, 5604 (2018).

[57] T. Wang, R. Cao, B. Ning, A. J. Dixon, J. A. Hossack, A. L. Klibanov, Q. Zhou, A. Wang, and S. Hu, Appl. Phys. Lett. 107, 153702 (2015).

[58] K. C. Balram, M. I. Davanço, B. R. Ilic, J.-H. Kyhm, J. D. Song, and K. Srinivasan, Phys. Rev. Applied 7, 024008 (2017).

[59] G. Soav, I. Tempra, M. F. Pantano, A. Cattoni, S. Collin, P. Biagioni, N. M. Pugno and G. Cerullo, ACS Nano 10, 2251 (2016).

[60] M. Li, H. X. Tang and M. L. Roukes, Nature Nanotech 2, 114 (2007).

[61]I. M. Sopko and G. A. Knyazev, Phys. Wave Phenom. 24, 124 (2016).

[62] D. Ahmed, X. Peng, A. Ozcelik, Y. Zheng and T. J. Huang, AIP Advances 5, 097161 (2015). 